\title{
A Fractional Anomalous Diffusion Model and Numerical Simulation for Sodium Ion Transport in the Intestinal Wall
}

\author{
Bo Yu and Xiaoyun Jiang \\ School of Mathematics, Shandong University, Jinan 250100, China \\ Correspondence should be addressed to Xiaoyun Jiang; wqjxyf@sdu.edu.cn
}

Received 17 May 2013; Accepted 1 July 2013

Academic Editor: Changpin Li

Copyright (C) 2013 B. Yu and X. Jiang. This is an open access article distributed under the Creative Commons Attribution License, which permits unrestricted use, distribution, and reproduction in any medium, provided the original work is properly cited.

\begin{abstract}
The authors present a fractional anomalous diffusion model to describe the uptake of sodium ions across the epithelium of gastrointestinal mucosa and their subsequent diffusion in the underlying blood capillaries using fractional Fick's law. A heterogeneous two-phase model of the gastrointestinal mucosa is considered, consisting of a continuous extracellular phase and a dispersed cellular phase. The main mode of uptake is considered to be a fractional anomalous diffusion under concentration gradient and potential gradient. Appropriate partial differential equations describing the variation with time of concentrations of sodium ions in both the two phases across the intestinal wall are obtained using Riemann-Liouville space-fractional derivative and are solved by finite difference methods. The concentrations of sodium ions in the interstitial space and in the cells have been studied as a function of time, and the mean concentration of sodium ions available for absorption by the blood capillaries has also been studied. Finally, numerical results are presented graphically for various values of different parameters. This study demonstrates that fractional anomalous diffusion model is appropriate for describing the uptake of sodium ions across the epithelium of gastrointestinal mucosa.
\end{abstract}

\section{Introduction}

The intestinal wall represents a complex system which allows the passage of substances either through the cells or in between the cells. The luminal surface of the intestine is covered with a typically leaky epithelium which enables the passage of ions via the intercellular route. The substance to be absorbed either penetrates into the intercellular space directly through the tight junction or enters the cell cytoplasm through the apical plasma membrane from the lumen of the intestine and then penetrates through the lateral plasma membrane to enter the intercellular space. The latter route leads to the underlying lamina propria, which consists of connective tissue, blood vessels, and lymph capillaries, and thus the substance enters the circulation (Figure 1) [1]. The process in which the ions enter the cell is passive diffusion under concentration gradient and potential gradient. This is mainly because transmural electrical potential differences of $5-12 \mathrm{mV}$ have been reported from a variety of species during recent years $[2,3]$. Although the potential differences across the intestinal wall are relatively small, they cannot be ignored in the studies of the intestinal transport of charged species [4].

Numerous techniques involving both in vivo and in vitro preparations have been employed in the study of intestinal transport. But because the cells are too small to provide continuous sections large enough for steady-state determinations of their transmission properties in actual physical situations, the distribution of the ions in the cellular and extracellular phases cannot be determined experimentally. Therefore, the idea of analysing such physiological problems using a theoretical approach has arisen. Fadali et al. [5] proposed an analytical model for water absorption in the intestine based on an integration of mass balance equation for active contact area for absorption. The model gave a solution for the amount of water absorbed in the intestine as a function of time following water ingestion using data from the physiological literature. Hills [6] proposed a two-phase model to study linear bulk diffusion into a continuous fluid 


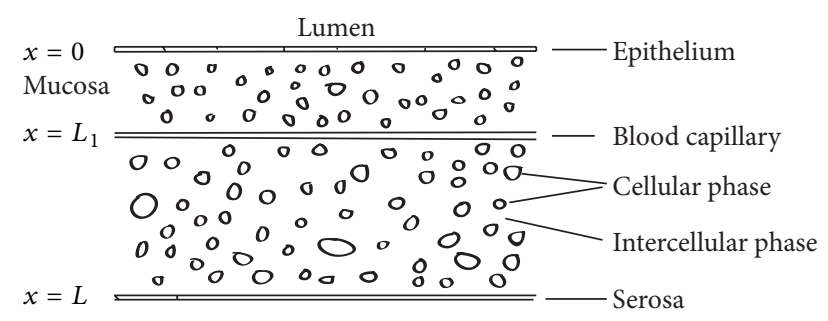

FIGURE 1: Schematic diagram of the intestinal wall.

in which a less permeable phase was distributed as particles of irregular profile; the overall uptake of solute by a parallelfaced section of tissue could be expressed as the sum of an infinite number of exponential terms. The advantage of this model was that it represented the histology of cellular tissue in the most realistic manner. Karmakar and Jayaraman [1] presented a linear diffusion model of the intestinal wall to describe the uptake of lead ions across the epithelium of gastrointestinal mucosa and their subsequent diffusion in the underlying blood capillaries. The model studied the variation of concentration with time in the extracellular phase and the cellular phase and the mean concentration available for absorption by the blood capillaries as a function of time, and it also reported the determination of membrane permeability for lead through theoretical analysis. Varadharajan and Jayaraman [7] presented a theoretical approach to study the uptake of sodium ions across the gastrointestinal mucosa and the concentrations at which they were taken up into the underlying blood capillaries. The model took into account both the diffusion under concentration gradient and potential gradient and active transport which was ATPase enzyme mediated, and appropriate partial differential equations for the two mechanisms of transport had been derived and were solved by iterative methods.

Recently, fractional calculus has been a subject of worldwide attention due to its surprisingly broad range of applications in physics, chemistry, engineering, economics, biology, and so forth [8-11]. In particular, fractional calculus is a key tool to study anomalous diffusion in transport processes which implies a fractional Fick's law for the flux that accounts for spatial and temporal nonlocality. Many literatures have shown that the power-law behavior is a hallmark of many biological phenomena observed at different scales and at various levels of organization and that a rheological behavior that conforms to the power law can be described by using methods of fractional calculus [12-16]. For example, Magin et al. [12] describe the formulation of the bioheat transfer in one dimension in terms of the fractional order differentiation with respect to time. His study demonstrates that fractional calculus can provide a unified approach to examine the periodic heat transfer in peripheral tissue regions. In this paper, according to Magin's idea, based on the previous analysis and fractional calculus theory, we consider a fractional anomalous diffusion model to describe the uptake of sodium ions across the epithelium of gastrointestinal mucosa using fractional Fick's law. In Section 2, we present the fractional anomalous diffusion model, and appropriate partial differential equations describing the variation with time of concentrations of sodium ions in both the interstitial phase and the intracellular phase across the intestinal wall are obtained using Riemann-Liouville space-fractional derivative and are solved by numerical computation. In Section 3, numerical results are presented graphically for various values of different parameters. In Section 4, we have presented our conclusions.

\section{Materials and Methods}

The fractional anomalous diffusion model considers a twophase structure of the intestinal wall in which the epithelium is treated as a thin layer. The apical plasma membrane is adjacent to the lumen of the intestine and at the origin of a one-dimensional coordinate system. The rest of the cellular elements form a uniformly distributed array of identical cells. In between are the intercellular spaces which correspond to interstitial phase (Figure 1).

2.1. Fractional Fick's Law. Fick's law is extensively adopted as a model for standard diffusion processes. For example, the simplest reaction diffusion model in spherical coordinates can be expressed as

$$
\frac{\partial C(r, t)}{\partial t}=-\frac{1}{r^{2}} \frac{\partial\left(r^{2} J(r, t)\right)}{\partial r}+f(r, t),
$$

where $C(r, t)$ is the concentration of solute (with radial symmetry), $f(r, t)$ represents reaction kinetics, and $J(r, t)$ is dispersive flux. Generally, Fick's law is used in normal diffusion for dispersive flux based on empirical observations:

$$
J(r, t)=-D \frac{\partial C(r, t)}{\partial r},
$$

where $D$ is the diffusion coefficient.

However, requiring separation of scales, it is not suitable for describing nonlocal transport process. In order to study the anomalous diffusion, the fractional Fick's law has been proposed [17], where the gradient of the solute concentration in the empirical flux equation is replaced by a fractional-order derivative:

$$
J(r, t)=-D \frac{\partial^{1-\lambda}}{\partial t^{1-\lambda}}\left(\frac{\partial^{\alpha-1} C(r, t)}{\partial r^{\alpha-1}}\right),
$$

where $0<\lambda \leq 1,1<\alpha \leq 2$, and $D$ is the anomalous diffusion coefficient. $\partial^{1-\lambda} / \partial t^{1-\lambda}$ and $\partial^{\alpha} / \partial r^{\alpha}$ are RiemannLiouville operators which are defined as follows:

$$
\begin{gathered}
\frac{\partial^{1-\lambda} C(r, t)}{\partial t^{1-\lambda}}=\frac{1}{\Gamma(\lambda)} \frac{\partial}{\partial t} \int_{0}^{t} \frac{C(r, \tau)}{(t-\tau)^{1-\lambda}} d \tau, \\
\frac{\partial^{\alpha} C(r, t)}{\partial r^{\alpha}}=\frac{1}{\Gamma(2-\alpha)} \frac{\partial^{2}}{\partial r^{2}} \int_{0}^{r} \frac{C(\tau, t)}{(r-\tau)^{\alpha-1}} d \tau,
\end{gathered}
$$

where $0<\lambda \leq 1,1<\alpha \leq 2$. We name it as the time-space fractional Fick's law [17]. Some special cases of this equation 
are as follows when $\lambda=1, \alpha=2$, it gives the classical Fick's law; when $\alpha=2$, it gives time fractional Fick's law; when $\lambda=$ 1 , it gives space fractional Fick's law. Here, we only consider the case of $\lambda=1$, that is, the space fractional Fick's law.

2.2. Fractional Anomalous Diffusion Model. The diffusion of sodium ions is complicated because its flux is determined by both the concentration gradient and the electrical gradient. Considering the motion of sodium ions under all forces, Macey [18] proposes that the flux equation is written as follows:

$$
J=-D\left(\frac{\partial C}{\partial x}+\frac{Z F}{R T} \frac{C \partial \psi}{\partial x}\right)
$$

where $D$ is the diffusion coefficient, $\psi$ is the electrical potential, $C$ is the concentration of the sodium ions, $x$ is the distance across the wall measured from the lumen, $Z$ is the charge on the ion ( +1 for the sodium ion), $F$ is Faraday's constant $\left(96500 \mathrm{C} \mathrm{mol}^{-1}\right), R$ is the universal gas constant, and $T$ is the absolute temperature.

Here, according to Magin's idea [12], based on the space fractional Fick's law, the flux equation is expressed in the following form:

$$
J=-D_{\alpha}\left(\frac{\partial^{\alpha-1} C}{\partial x^{\alpha-1}}+\frac{Z F}{R T} \frac{C \partial \psi}{\partial x}\right)
$$

where $1<\alpha \leq 2, D_{\alpha}$ is the anomalous diffusion coefficient. The first term on the right stands for the concentration gradient, and the second term on the right stands for the electrical gradient.

We consider a two-phase model consisting of the interstitial phase and the intracellular phase. The mass balance equation in the interstitial phase, which accounts for the molecular diffusion flux and a uniformly distributed continuum of point sinks whose strength is proportional to the local concentration differences between the two phases [1], is

$$
\frac{\partial C_{1}^{\prime}}{\partial t}=-\nabla \cdot J+P\left(C_{2}^{\prime}-C_{1}^{\prime}\right)
$$

where $C_{1}^{\prime}$ and $C_{2}^{\prime}$ are the concentrations of sodium ions in the interstitial phase and in the intracellular phase, respectively, and $P$ is the membrane permeability coefficient for the molecular diffusion of sodium ions into the cellular phase. Substituting (6) into (7), we can get the following equation:

$$
\begin{aligned}
\frac{\partial C_{1}^{\prime}}{\partial t}= & D_{\alpha}\left[\frac{\partial^{\alpha} C_{1}^{\prime}}{\partial x^{\alpha}}+\frac{Z F}{R T}\left(\frac{\partial C_{1}^{\prime}}{\partial x} \frac{\partial \psi}{\partial x}+\frac{\partial^{2} \psi}{\partial x^{2}} C_{1}^{\prime}\right)\right] \\
& +P\left(C_{2}^{\prime}-C_{1}^{\prime}\right) .
\end{aligned}
$$

And based on the assumption that diffusion does not contribute significantly to the total molecular transport inside the cell [1], the mass balance equation in the cellular phase is

$$
\frac{\partial C_{2}^{\prime}}{\partial t}=P\left(C_{1}^{\prime}-C_{2}^{\prime}\right)
$$

which is justified by the fact that the dimensions of the cells are small compared to the thickness of the intestinal wall; therefore, the flux through them is independent of distance.

Meanwhile, we assume that $\psi=A^{\prime} x$, where $A^{\prime}$ is a constant to be determined. A justification for the constant field assumption can be found in the observation that if a membrane contains a large number of dipolar ions close to their isoelectric point, these dipoles will tend to alter their orientation in such a way that they tend to smooth out any irregularities and maintain a constant field [7]. The validity of this assumption has also been discussed by Goldman [19] and Cole [20]. Hence, (8) can be reduced to

$$
\frac{\partial C_{1}^{\prime}}{\partial t}=D_{\alpha}\left(\frac{\partial^{\alpha} C_{1}^{\prime}}{\partial x^{\alpha}}+\frac{Z F}{R T} \frac{\partial C_{1}^{\prime}}{\partial x} A^{\prime}\right)+P\left(C_{2}^{\prime}-C_{1}^{\prime}\right)
$$

that is,

$$
\frac{\partial C_{1}^{\prime}}{\partial t}=D_{\alpha}\left(\frac{\partial^{\alpha} C_{1}^{\prime}}{\partial x^{\alpha}}+A \frac{\partial C_{1}^{\prime}}{\partial x}\right)+P\left(C_{2}^{\prime}-C_{1}^{\prime}\right),
$$

where $A=A^{\prime}(Z F / R T)$.

The value $x=0$ corresponds to the lumen of the intestine and $x=L$ corresponds to serosa. We are interested in finding the ion concentration at $x=L_{1}$, which corresponds to the blood capillary at which it is absorbed (Figure 1). In rats, the mucosal epithelium is approximately 0.14 of the total intestinal wall thickness. Equations (9) and (11) are solved to obtain $C_{1}^{\prime}$ and $C_{2}^{\prime}$ as functions of $x$ and $t$, and the mean concentration of sodium ions at $x=L_{1}$ is calculated from

$$
M C=\frac{\gamma_{1} C_{1}\left(L_{1}\right)+\gamma_{2} C_{2}\left(L_{1}\right)}{\gamma_{1}+\gamma_{2}},
$$

where $\gamma_{1}$ and $\gamma_{2}$ are the interstitial and intracellular volume fractions, respectively.

Then, we introduce dimensionless parameters

$$
\begin{array}{lll}
t^{*}=\frac{t D_{\alpha}}{L^{2}}, & x^{*}=\frac{x}{L}, & \beta=\frac{P L^{2}}{D_{\alpha}}, \\
C_{1}=\frac{C_{1}^{\prime}}{C_{L}^{\prime}}, & C_{2}=\frac{C_{2}^{\prime}}{C_{L}^{\prime}}, & L_{1}^{*}=\frac{L_{1}}{L},
\end{array}
$$

to reduce (11) and (9) to the nondimensional form (with the * notation dropped for convenience):

$$
\begin{gathered}
\frac{\partial C_{1}}{\partial t}=\frac{1}{L^{\alpha-2}} \frac{\partial^{\alpha} C_{1}}{\partial x^{\alpha}}+A \frac{\partial C_{1}}{\partial x}+\beta\left(C_{2}-C_{1}\right), \\
\frac{\partial C_{2}}{\partial t}=\beta\left(C_{1}-C_{2}\right),
\end{gathered}
$$

where $C_{L}^{\prime}$ is the concentration of sodium ions in the lumen. The parameter $\beta=P L^{2} / D$ could be considered as the ratio of the membrane diffusion flux into the cellular phase to the molecular diffusion flux in the interstitial phase.

Based on the assumption that the concentration of sodium ions in the intestinal lumina surface is equal to the 
concentration at its abluminal surface for the epithelial is specially thin, the boundary conditions are given by

$$
C_{1}(0, t)=1, \quad C_{1}(1, t)=0,
$$

which mean that the concentration of sodium ions in the lumen is set to 1 , whereas at the serosa it is set to 0 at all times. Further, the initial concentration is taken to be 0 , a condition justified in the case of in vitro experiments. Both $C_{1}$ and $C_{2}$ are set to 0 at any point within the tissue when time equals 0 . Therefore, the initial conditions are given by

$$
C_{1}(x, 0)=0, \quad C_{2}(x, 0)=0 .
$$

2.3. Numerical Computation. For the numerical solution of the problem above, we introduce a uniform grid of mesh points $\left(x_{j}, t_{k}\right)$, with $x_{j}=j h, j=0,1, \ldots, N$, and $t_{k}=$ $k \tau, k=0,1, \ldots, M$, where $M$ and $N$ are two positive integers, $h=1 / N$ and $\tau=T / M$ are the uniform spatial and temporal mesh size, respectively. The theoretical solution $C_{1}$ at the point $\left(x_{j}, t_{k}\right)$ is denoted by $C_{1}\left(x_{j}, t_{k}\right)$; the solution of an approximating difference scheme at the point $\left(x_{j}, t_{k}\right)$ will be denoted by $C_{1, j}^{k}$. Similarly, the theoretical solution $C_{2}$ at the point $\left(x_{j}, t_{k}\right)$ is denoted by $C_{2}\left(x_{j}, t_{k}\right)$; the solution of an approximating difference scheme at the point $\left(x_{j}, t_{k}\right)$ will be denoted by $C_{2, j}^{k}$.

Then, we start to introduce the discretization of the differential operators. The first-order derivatives with respect to the temporal variable $\partial C_{1} / \partial t$ and $\partial C_{2} / \partial t$ are approximated by the following Euler backward difference, respectively:

$$
\begin{aligned}
& \frac{\partial C_{1}\left(x_{j}, t_{k}\right)}{\partial t} \approx \frac{C_{1}\left(x_{j}, t_{k}\right)-C_{1}\left(x_{j}, t_{k-1}\right)}{\tau}, \\
& \frac{\partial C_{2}\left(x_{j}, t_{k}\right)}{\partial t} \approx \frac{C_{2}\left(x_{j}, t_{k}\right)-C_{2}\left(x_{j}, t_{k-1}\right)}{\tau},
\end{aligned}
$$

and the first-order derivative with respect to the spatial variable $\partial C_{1} / \partial x$ is approximated by Euler forward difference:

$$
\frac{\partial C_{1}\left(x_{j}, t_{k-1}\right)}{\partial x} \approx \frac{C_{1}\left(x_{j+1}, t_{k-1}\right)-C_{1}\left(x_{j}, t_{k-1}\right)}{h} .
$$

As for the Riemann-Liouville fractional derivative, using the relationship between the Grünwald-Letnikov formula and Riemann-Liouville fractional derivative, we can approximate the fractional derivative by $[21,22]$

$$
\frac{\partial^{\alpha} C_{1}\left(x_{j}, t_{k-1}\right)}{\partial x^{\alpha}} \approx h^{-\alpha} \sum_{l=0}^{j+1} \omega_{l}^{(\alpha)} C_{1}\left(x_{j}-(l-1) h, t_{k-1}\right)
$$

where $\omega_{0}^{(\alpha)}=1, \omega_{k}^{(\alpha)}=(-1)^{k}(\alpha(\alpha-1) \cdots(\alpha-k+1) / k !)$ for $k \geq 1$. There, we have adopted the shifted Grünwald-Letnikov formula for $1<\alpha \leq 2$.
Finally, the finite difference method for the above problem is given as follows:

$$
\begin{gathered}
\frac{C_{1, j}^{k}-C_{1, j}^{k-1}}{\tau}=\frac{h^{-\alpha}}{L^{\alpha-2}} \sum_{l=0}^{j+1} \omega_{l}^{(\alpha)} C_{1, j-l+1}^{k-1} \\
\quad+A \frac{C_{1, j+1}^{k-1}-C_{1, j}^{k-1}}{h}+\beta\left(C_{2, j}^{k-1}-C_{1, j}^{k-1}\right), \\
\quad k=1,2, \ldots, M, \quad j=1,2, \ldots, N-1, \\
\quad \frac{C_{2, j}^{k}-C_{2, j}^{k-1}}{\tau}=\beta\left(C_{1, j}^{k-1}-C_{2, j}^{k-1}\right), \\
k=1,2, \ldots, M, \quad j=0,1,2, \ldots, N-1, N .
\end{gathered}
$$

The boundary and initial conditions can be discretized by

$$
\begin{gathered}
C_{1,0}^{k}=1, \quad C_{1, N}^{k}=0, \quad k=0,1, \ldots, M, \\
C_{1, j}^{0}=0, \quad j=1,2, \ldots, N, \\
C_{2, j}^{0}=0, \quad j=0,1,2, \ldots, N .
\end{gathered}
$$

The concentrations of the sodium ions in the intercellular phase and intracellular phase are determined at different steps of time and space, and their weighted mean concentration at the blood capillaries can also be obtained.

\section{Results and Discussion}

The thickness of the intestinal wall $L$ is taken to be $2.14 \times$ $10^{-4} \mathrm{~m}[1]$, which is measured from a cross-section of the rat intestinal wall using an ocular micrometer fitted to a simple microscope. Muller [23] reported in morphometric studies of rat gastric mucosa that the epithelial cells occupied $74 \%$ while the remaining $26 \%$ was occupied by lamina propria. Hence, a choice of 0.26 is made for $\gamma_{1}$ and 0.74 for $\gamma_{2}$, arbitrarily as their values are not available in the literatures. According to $\mathrm{Xu}$ and Zhao [24], the permeability coefficient $P$ of sodium ions is taken to be $1.61 \times 10^{-5} \mathrm{~s}^{-1}$. Varadharajan and Jayaraman [7] had studied that the numerical value of $A$ depended on the potential difference between the serosa and the mucosa, and, comparing with the experimental results of Lauterbach [25], Varadharajan obtained the value of $A$ to be $0 \leq A \leq 1$ and the optimum value of $A$ to be around 0.4 , so $A$ is taken to be 0.4 in our studies.

3.1. The Anomalous Diffusion Coefficient $D_{\alpha}$ of Sodium Ions. According to the Stokes-Einstein formula $D=k T / 6 \pi \mu r$, where $k$ is Boltzmann's constant $\left(1.4 \times 10^{-23} \mathrm{JK}^{-1}\right)$, $T$ is the temperature $(\sim 310 \mathrm{~K}), \mu$ is the viscosity of intercellular fluid ( 0.001 Pas), and $r$ is the radius of the water molecule $(\sim 0.45 \mathrm{~nm})$, we can obtain the diffusivity in water $D=5.12 \times$ $10^{-10} \mathrm{~m}^{2} \mathrm{~s}^{-1}$ [1]. Nevertheless, it can also be considered as a reasonable approximation for the diffusion coefficient $D_{\alpha}$ of sodium ions. Here, we change this parameter to $0.3,0.7,1$, 1.3 , and 1.7 times of the value of diffusivity $D$. 


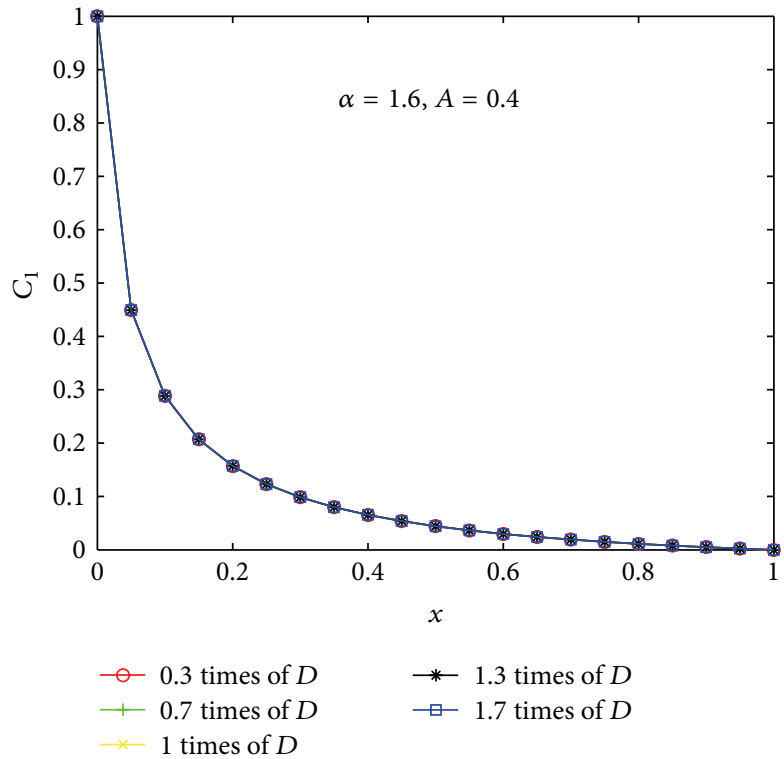

(a)

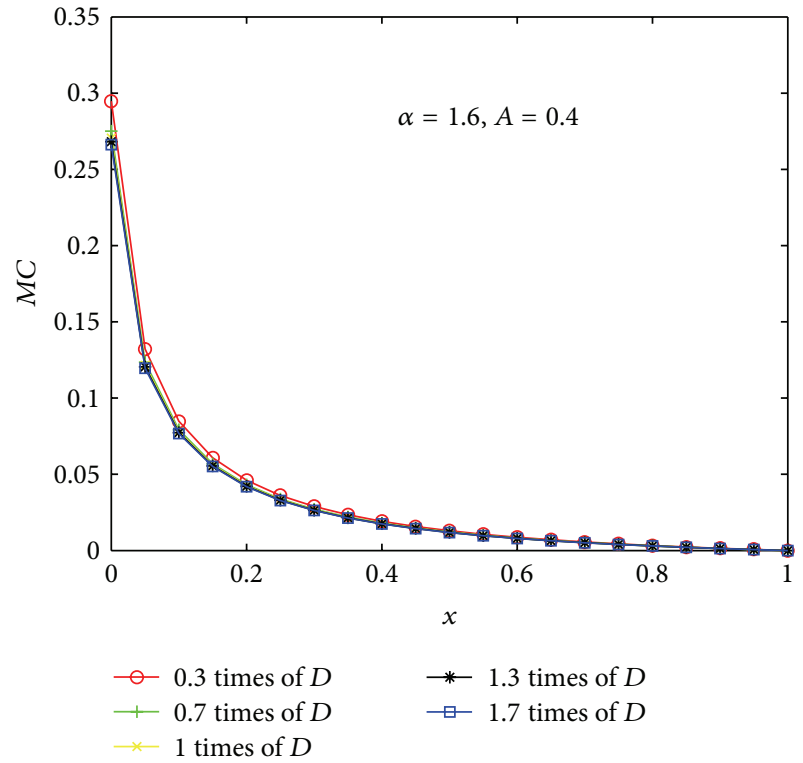

(b)

Figure 2: (a) is $C_{1}$, plotted against $x$ for different values of $D_{\alpha}$ when $\alpha=1.6$; (b) is $M C$, plotted against $x$ for different values of $D_{\alpha}$ when $\alpha=1.6$.

Figure 2, individual graph, demonstrates the variation of concentration with respect to $x$ for different values of $D_{\alpha}$ when $\alpha=1.6$. During the course of diffusion, we find that the concentrations decrease in an exponential way, and the curves tend to change very little when the values of $D_{\alpha}$ vary not too much, which indicates that the distribution of sodium ions is more uniform; therefore, we can consider any of these values as a reasonable approximation for the diffusion coefficient $D_{\alpha}$ of sodium ions. By amplifying the figures, we also observe that smaller $D_{\alpha}$ increases the amplitude of $M C$, which indicates that larger $D_{\alpha}$ increases the speed of the absorption especially at the blood capillaries, a possible explanation is that faster movement of sodium ions makes it easier for diffusion.

3.2. The Order $\alpha$ of Fractional Derivative. Here, based on the above analysis, we take $D_{\alpha}$ to be $0.38 \times 10^{-5} \mathrm{~cm}^{2} \mathrm{~s}^{-1}$, which is about 0.7 times of the diffusivity $D$, just as used in the literature [18]. Substituting the respective values of $D_{\alpha}$ and $L$, we can obtain that the actual time is about $2 \min \times t$.

Figure 3 demonstrates that the variation of concentration with respect to $x$ at $t=20 \mathrm{~min}$ for different values of $\alpha$. Obviously, we can observe that the concentrations decrease in an exponential way and the curves become smoother as the value of $\alpha$ becomes larger, which indicate that the diffusion in the intercellular phase and the absorption especially at the blood capillaries become quicker as the value of $\alpha$ becomes smaller. This fact demonstrates that the diffusion of sodium ions is anomalous superdiffusion.
Figure 4 demonstrates that the variation of concentration with respect to $x$ at different times when $\alpha=1.6$, $A=0.4$. During the course of diffusion, we find that the concentrations decrease in an exponential way. By amplifying the figures, we obviously observe that the decay tends to be smoother and smoother when time increases, which indicates the distribution of sodium ions is more uniform. Figure 4(a) shows that at earlier times most of the sodium ions are absorbed by the cells, and for later times they tend to pass towards the serosa. Meanwhile, we observe that most of the obsorption takes place at $x<0.2$ from Figure 4(b), which can be explained as the distance at which the blood capillaries lie. This is quite reasonable, since in rats the mucosal epithelium is about 0.14 of the total wall thickness [1]. All these phenomena are connective with the results of Varadharajan and Jayaraman [7], which indicate that the anomalous diffusion is appropriate for describing the uptake of sodium ions.

Figure 5(a) is $M C$ plotted against $t$ at different $x$ when $\alpha=1.9, A=0.4$. We find that, at a particular distance, $M C$ increases with time, but at a farther distance, the flux is lower because the major absorption is made available at the distance where the blood capillaries lie. Figure 5(b) is $M C$ plotted against $t$ at $x=0.15$ for different values of $\alpha$. We find that, at a particular distance $x=0.15, M C$ is lower when $\alpha$ is smaller, which indicates that the diffusion is quicker when the value of $\alpha$ is smaller, and this leads to the lower value of $M C$.

Figure 6(a) is $C_{1}$, plotted against $t$ at $x=0.15$ for different values of $\alpha$. We find that, at a particular distance $x=0.15$, 


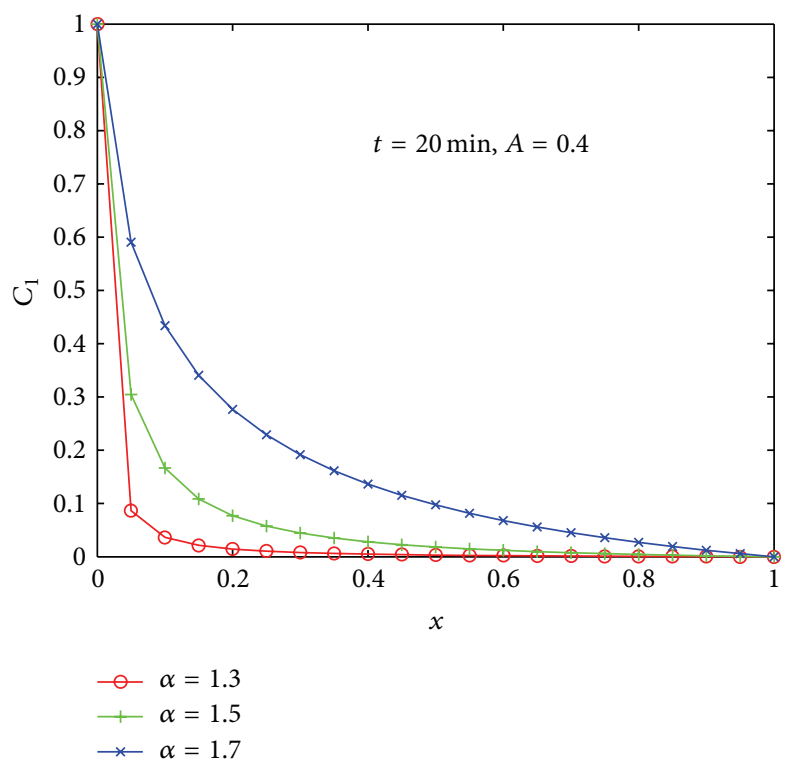

(a)

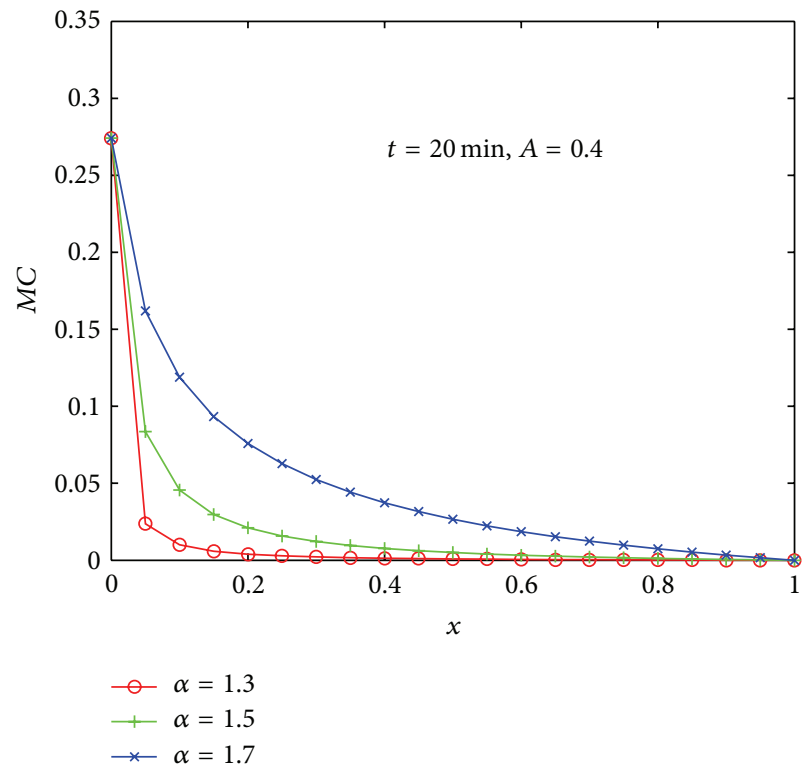

(b)

Figure 3: (a) is $C_{1}$, plotted against $x$ at $t=20 \mathrm{~min}$ for different values of $\alpha$; (b) is $M C$, plotted against $x$ at $t=20$ min for different values of $\alpha$.

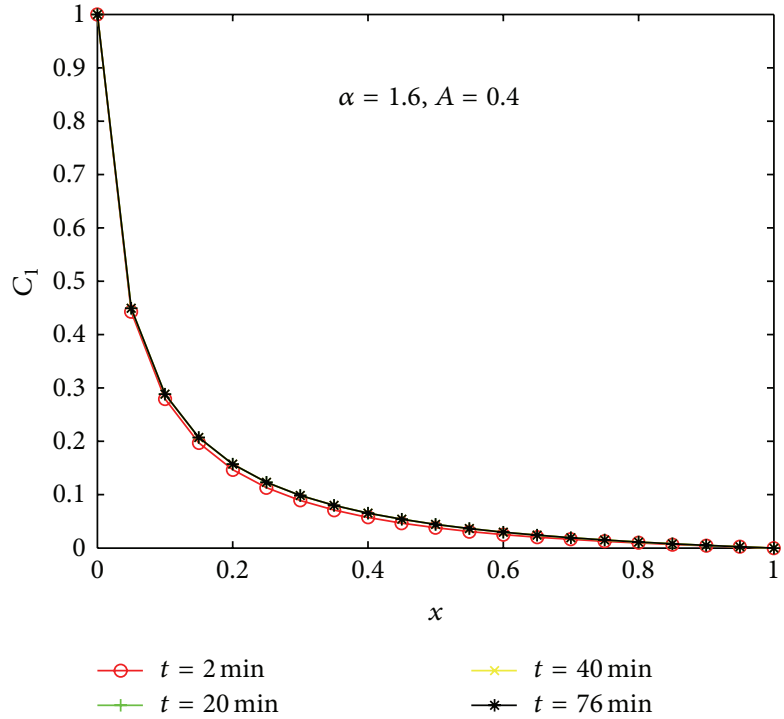

(a)

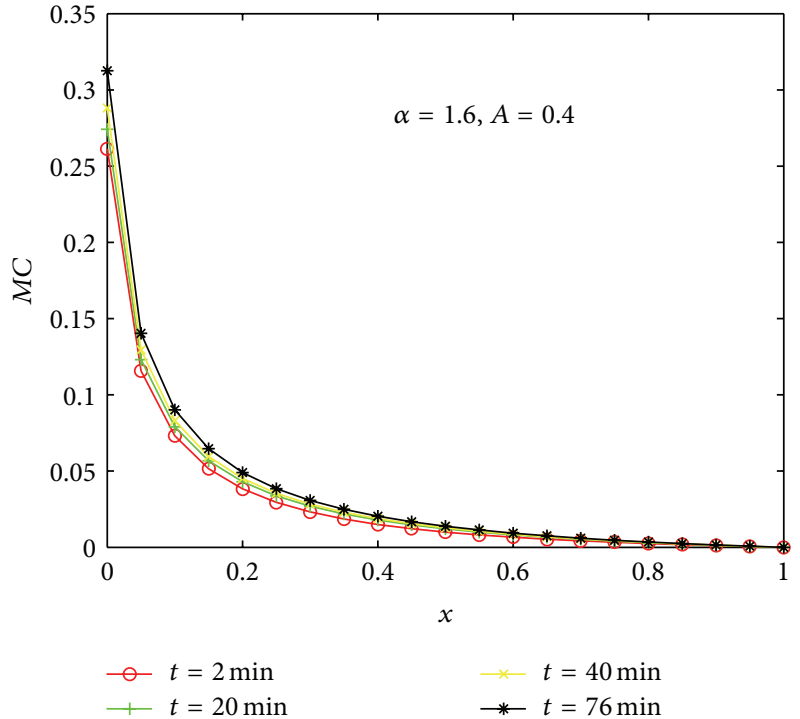

(b)

Figure 4: (a) is $C_{1}$, plotted against $x$ at different times when $\alpha=1.6, A=0.4$; (b) is $M C$, plotted against $x$ at different times when $\alpha=1.6$, $A=0.4$.

$C_{1}$ is lower when $\alpha$ is smaller, which indicates that the diffusion is quicker when the value of $\alpha$ is smaller, and this leads to the lower value of $C_{1}$. Figure 6(b) is $C_{1}$ plotted against $t$ at different $x$ when $\alpha=1.96, A=0.4$. We find that about $23 \%$ sodium ion absorption is achieved at a distance of $0.1-0.15$ when we choose $\alpha=1.96$, and it is in good agreement with the experimental results of Lauterbach [25], which indicate that the concentration of $\mathrm{Na}$ in the cell water approaches $23 \%$ of the initial concentration of the incubation medium after the addition to the luminal side. 


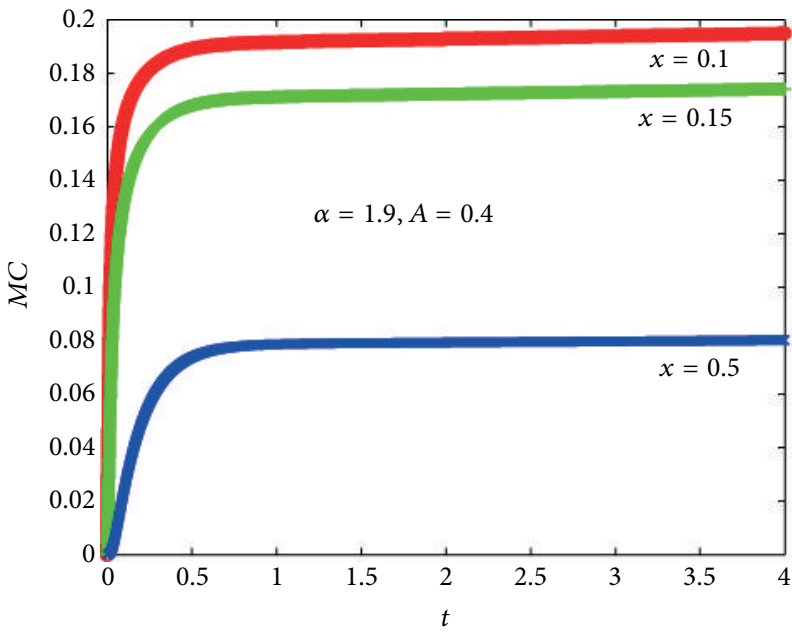

(a)

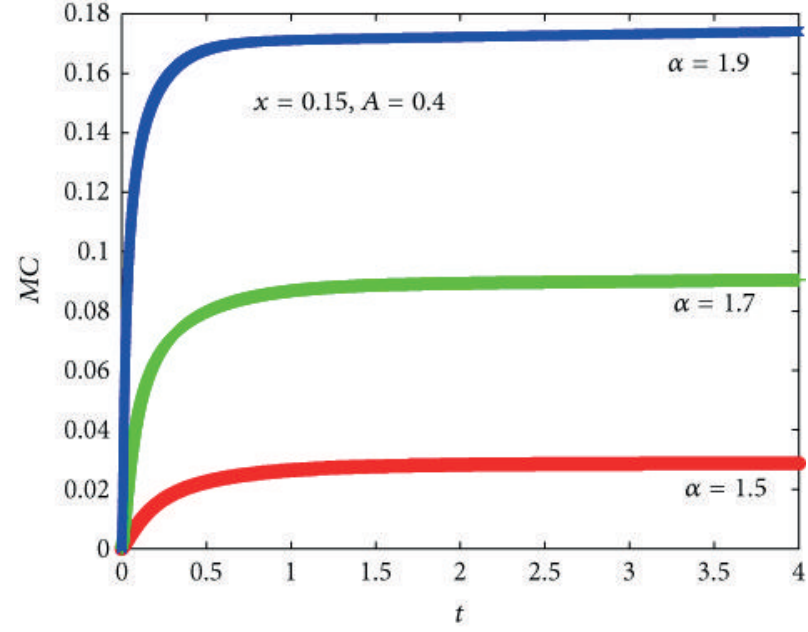

(b)

FIGURE 5: (a) is $M C$, plotted against $t$ at different $x$ when $\alpha=1.9, A=0.4$; (b) is $M C$, plotted against $t$ at $x=0.15$ for different values of $\alpha$.

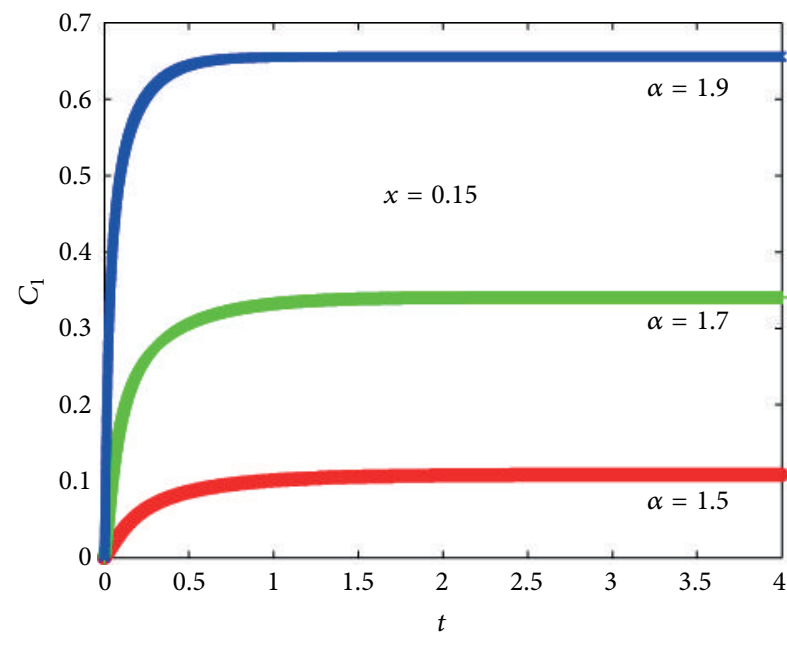

(a)

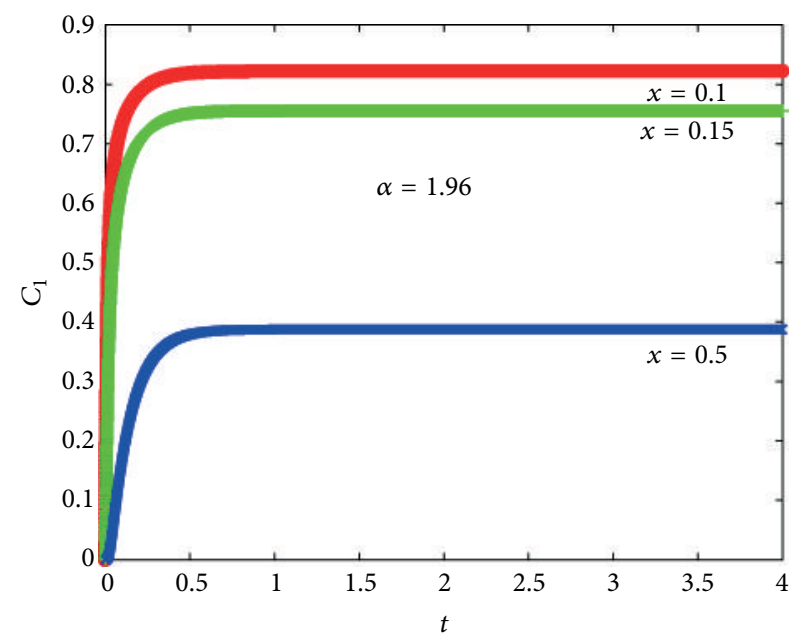

(b)

Figure 6: (a) is $C_{1}$, plotted against $t$ at $x=0.15$ for different values of $\alpha$; (b) is $C_{1}$, plotted against $t$ at different $x$ when $\alpha=1.96, A=0.4$.

\section{Conclusions}

In summary, in this paper we have derived a fractional anomalous diffusion model for sodium ion transport in the intestinal wall using space fractional Fick's law. Appropriate partial differential equations describing the variation with time of concentrations of sodium ions in both the interstitial phase and the intracellular phase across the intestinal wall are obtained using Riemann-Liouville space-fractional derivatives and are solved by finite difference methods. The numerical simulations have been discussed, and numerical results are presented graphically for various values of different parameters. It demonstrates that fractional anomalous diffusion model is appropriate for describing the uptake of sodium ions across the epithelium of gastrointestinal mucosa. This research also provides some new points for studying ions transferring processes in biological systems.

\section{Acknowledgment}

The project was supported by the National Natural Science Foundation of China (Grants nos. 11072134, 11102102, and 91130017). 


\section{References}

[1] N. Karmakar and G. Jayaraman, "Linear diffusion of lead in the intestinal wall: A Theoretical Study," IMA Journal of Mathematics Applied in Medicine and Biology, vol. 5, no. 1, pp. 33-43, 1988.

[2] W. M. Armstrong, "Cellular mechanisms of ion transport in the small intestine," Physiology of the Gastrointestinal Tract 2, pp. 1251-1266, 1987.

[3] S. G. Schultz, "Principles of electrophysiology and their application to epithelial tissues, gastrointestinal physiology," in Gastrointestinal Physiology, pp. 87-91, Butterworth, London, UK, 1974.

[4] S. G. Schultz and P. F. Curran, Handbook of Physiology, vol. 3, Section 6, 1968.

[5] M. S. Fadali, J. W. Steadman, and R. G. Jacquot, "A model of water absorption in human intestine," Biomedical Sciences Instrumentation, vol. 16, pp. 49-53, 1980.

[6] B. A. Hills, "Linear bulk diffusion into heterogeneous tissue," The Bulletin of Mathematical Biophysics, vol. 30, no. 1, pp. 47-59, 1968.

[7] M. Varadharajan and G. Jayaraman, "Sodium ion transport in the intestinal wall: a mathematical model," IMA Journal of Mathematical Medicine and Biology, vol. 11, no. 3, pp. 193-205, 1994.

[8] D. Baleanu, A. K. Golmankhaneh, R. Nigmatullin, and A. K. Golmankhaneh, "Fractional Newtonian mechanics," Central European Journal of Physics, vol. 8, no. 1, pp. 120-125, 2010.

[9] J. Singh, P. K. Gupta, and K. N. Rai, "Solution of fractional bioheat equations by finite difference method and HPM," Mathematical and Computer Modelling, vol. 54, no. 9-10, pp. 2316-2325, 2011.

[10] R. Metzler and J. Klafter, “The random walk's guide to anomalous diffusion: a fractional dynamics approach," Physics Reports, vol. 339, no. 1, pp. 1-77, 2000.

[11] R. Metzler and J. Klafter, "The restaurant at the end of the random walk: recent developments in the description of anomalous transport by fractional dynamics," Journal of Physics A, vol. 37, no. 31, pp. R161-R208, 2004.

[12] R. L. Magin, S. C. Boregowda, and C. Deodhar, "Modeling of pulsating peripheral bio-Heat transferusing fractional calculus and constructal theory," International Journal of Design, Nature, and Ecodynamics, vol. 1, no. 18, pp. 18-33, 2007.

[13] X. Y. Jiang and H. T. Qi, "Thermal wave model of bioheat transfer with modified Riemann-Liouville fractional derivative," Journal of Physics, vol. 45, no. 48, 10 pages, 2012.

[14] W. C. Tan, C. Fu, C. Fu, W. Xie, and H. Cheng, "An anomalous subdiffusion model for calcium spark in cardiac myocytes," Applied Physics Letters, vol. 91, no. 18, 3 pages, 2007.

[15] R. G. Larson, The Structure and Rheology of Complex Fluids, vol. 2, Oxford University Press, New York, NY, USA, 1999.

[16] R. L. Magin, "Fractional calculus models of complex dynamics in biological tissues," Computers \& Mathematics with Applications, vol. 59, no. 5, pp. 1586-1593, 2010.

[17] X. Y. Jiang, M. Y. Xu, and H. T. Qi, "The fractional diffusion model with an absorption term and modified Fick's law for non-local transport processes," Nonlinear Analysis: Real World Applications, vol. 11, no. 1, pp. 262-269, 2010.

[18] R. I. Macey, Membrane Physiology, Plenum, New York, NY, USA, 2nd edition, 1980.
[19] D. E. Goldman, "Potential, impedence, and rectification in membranes," The Journal of General Physiology, vol. 27, no. 1, pp. 37-60, 1943.

[20] K. S. Cole, "Electrodiffusion models for the membrane of squid giant axon," Physiological Reviews, vol. 45, pp. 340-379, 1965.

[21] M. M. Meerschaert and C. Tadjeran, "Finite difference approximations for fractional advection-dispersion flow equations," Journal of Computational and Applied Mathematics, vol. 172, no. 1, pp. 65-77, 2004.

[22] C. Li and F. Zeng, "Finite difference methods for fractional differential equations," International Journal of Bifurcation and Chaos in Applied Sciences and Engineering, vol. 22, no. 4, 2012.

[23] O. Muller, "The quantitation of the rat gastric mucosa by morphometric methods," Scandinavian Journal of Gastroenterology, Supplement, vol. 101, no. 1, pp. 1-6, 1984.

[24] M. Y. Xu and G. L. Zhao, "Nonisotonic reabsorption of water and steady state distribution of sodiumion in renal descending limb of Henle," Acta Mechanica Sinica, vol. 1, no. 3, 1986.

[25] F. O. Lauterbach, Intestinal Transport, University Park Press, 1976. 


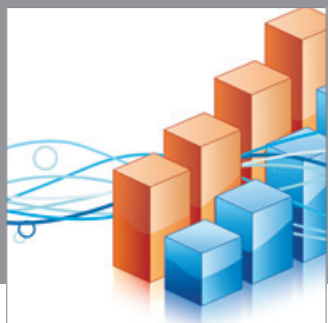

Advances in

Operations Research

mansans

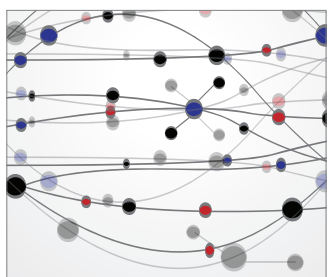

The Scientific World Journal
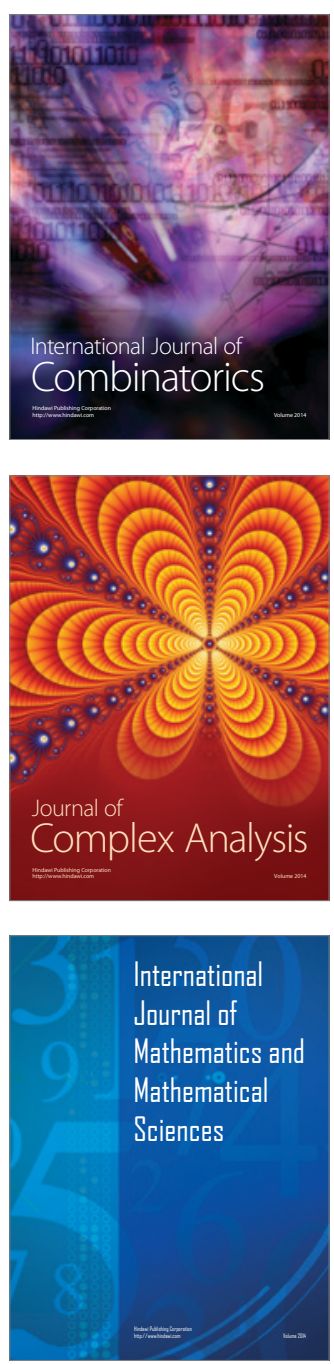
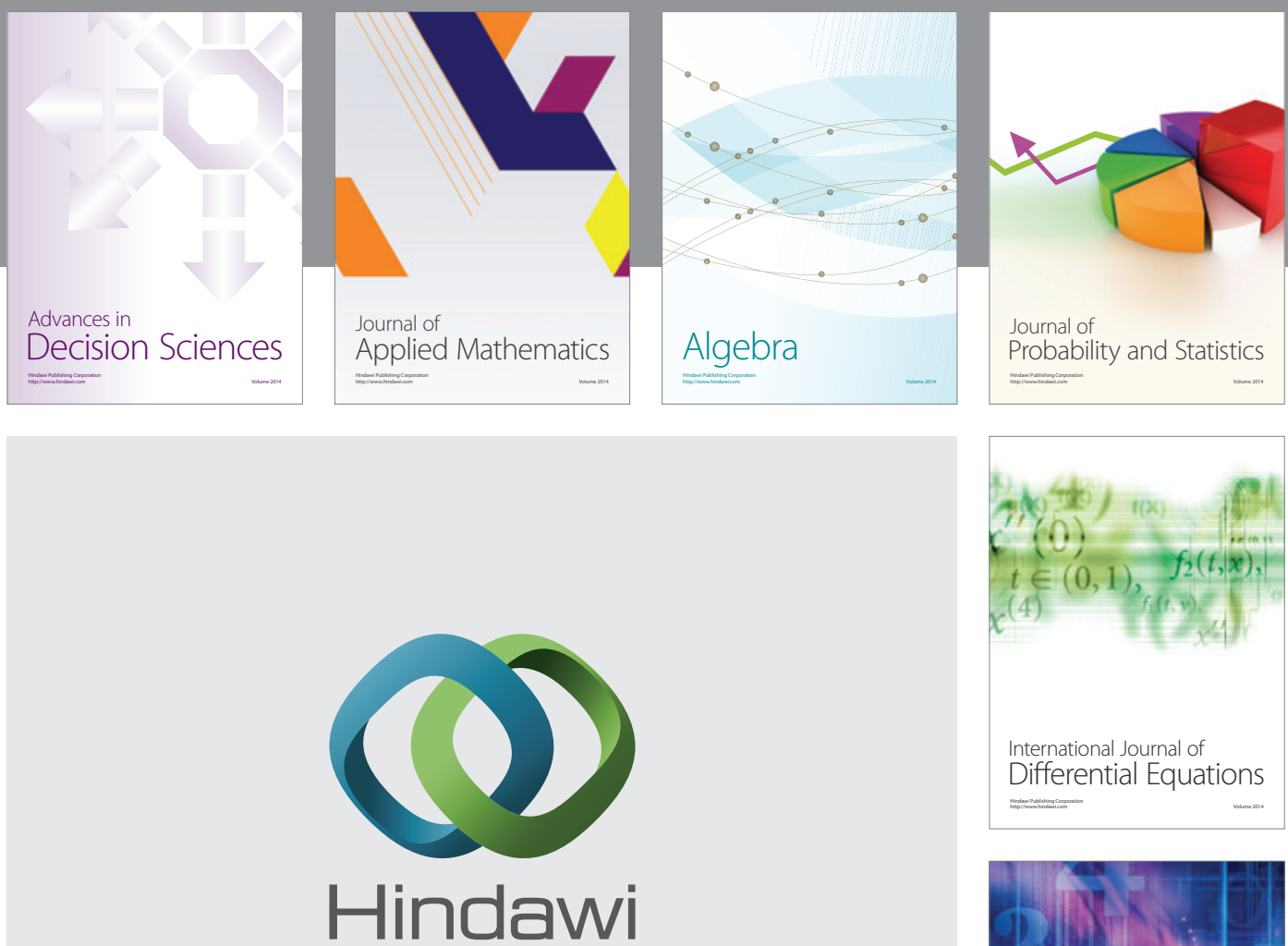

Submit your manuscripts at http://www.hindawi.com
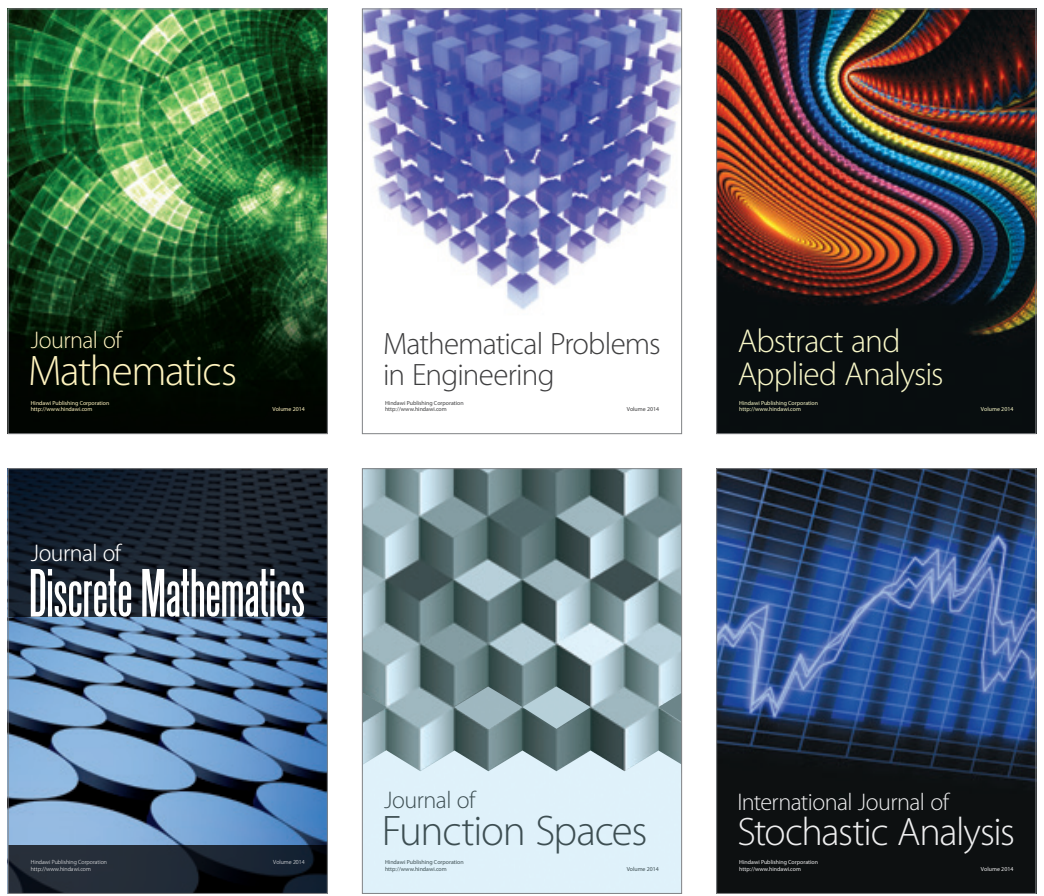

Journal of

Function Spaces

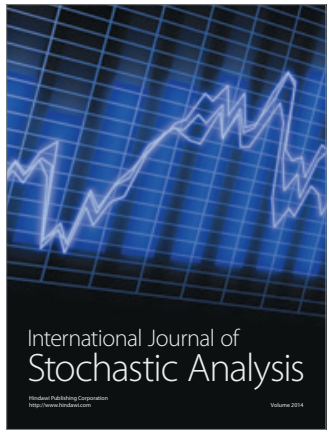

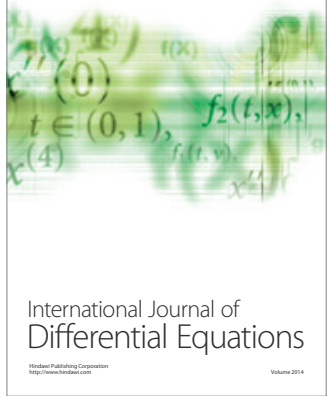
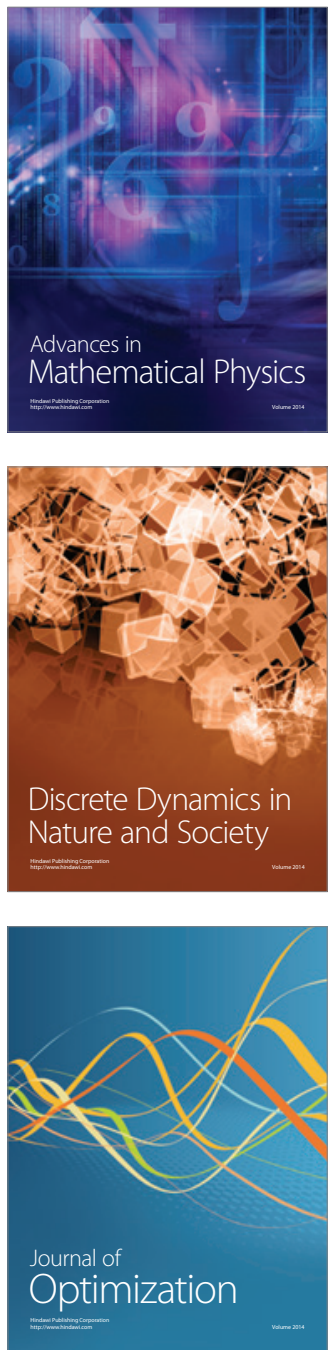